\title{
ESTIMATION OF THE POPULATION SUSCEPTIBILITY AGAINST MEASLES IN SLOVAKIA
}

\author{
Jana Zibolenová1, Zuzana Chladná ${ }^{1}$, Viera Švihrováa ${ }^{1}$, Tibor Baška ${ }^{1}$, Iveta Waczulíková ${ }^{3}$, Henrieta Hudečková1 \\ ${ }^{1}$ Department of Public Health, Jessenius Faculty of Medicine in Martin, Comenius University in Bratislava, Martin, Slovakia \\ ${ }^{2}$ Department of Applied Mathematics and Statistics, Faculty of Mathematics, Physics and Informatics, Comenius University, Bratislava, Slovakia \\ ${ }^{3}$ Division of Biomedical Physics, Department of Nuclear Physics and Biophysics, Faculty of Mathematics, Physics and Informatics, Comenius \\ University, Bratislava, Slovakia
}

\section{SUMMARY}

Objective: In Slovakia, thanks to a highly effective vaccination programme, no domestic cases of measles have been reported since 1999. However, there are several outbreaks of measles currently hitting some countries in Europe. Difficulties in reaching the goal of measles elimination make it necessary to monitor the status of the population susceptibility to prevent similar outbreaks in the future. We hypothesize that immunity wanes overtime, which can substantially impact the population susceptibility. This work introduces a model that estimates a proportion of individuals susceptible to measles in the Slovak population in 2015.

Methods: Our analysis is based on an age-cohort model that incorporates waning immunity, vaccination schedule and changes in demographic structure. The inputs of the model are data on the vaccination coverage, last seroprevalence survey in 2002 and age structure of the population.

Results: In a short-term horizon, waning immunity does not affect the estimated proportion of the susceptible population. However, in a longterm horizon, the antibody titers can fall below the level of protection, which would result in a substantial transfer of initially immune individuals to the compartment of the susceptible ones. Incorporating of waning immunity in the cohort model has indicated that the most susceptible cohorts are not-vaccinated youngest children and cohorts born between 1969 and 1986.

Conclusions: Applying the model to the current situation shows that people aged $30-45$ years and unvaccinated infants represent the most susceptible groups. Model partially replaces missing seroprevalence survey, but, because the parameters of model and phenomenon of waning immunity are not exactly known, we suggest reintroducing the regular national serosurveys in order to empirically determine the level of susceptibility for measles in Slovakia.

Key words: measles, waning immunity, cohort model, vaccination, susceptibility

Address for correspondence: J. Zibolenová, Department of Public Health, Jessenius Faculty of Medicine in Martin, Comenius University in Bratislava, Malá Hora 11149/4B, 03601 Martin, Slovak Republic. E-mail: jana.zibolenova@gmail.com

https://doi.org/10.21101/cejph.a4914

\section{INTRODUCTION}

Measles is a highly contagious, exanthematous disease. It is caused by morbillivirus within the family Paramyxoviridae (1). Measles had been considered one of the most frequent childhood diseases in the past. Since the introduction of mass vaccination in 1969 a permanent decline in incidence has been observed, which has been only interrupted by occasional outbreaks. No endemic and only few imported cases have been reported in Slovakia since1999 until the present time.

Vaccination by attenuated vaccine induces both, humoral and cellular responses. Even if these responses are of lower magnitude and shorter duration compared to those following wild-type measles virus infection, the antibody titers remain still sufficiently high to provide effective protection (1). However, recent research findings suggest that measles vaccine protection wanes with time, creating a real concern that proportion of individuals in a cohort who retain protective antibody levels following vaccination might be compromised over time. Thus, the effect of waning immunity could potentially lead to an increase of susceptible individuals in the population concerned (2-9).

Despite the fact that in the recent years measles cases have not occurred in Slovakia, it still remains a threat to the current or near-future population. Since the last national seroprevalence survey was performed in 2002, the current status of the population susceptibility to measles is uncertain. The aim of this work is to build a model of waning immunity and incorporate it into an age cohort model of the Slovak population in order to estimate the fraction of individuals susceptible to measles in 2015.

\section{MATERIALS AND METHODS}

Our approach is based on a cohort model. By the term cohort we understand a group of individuals born in the same year. We assume that the Slovak population can be divided into 100 age cohorts. The inputs of our model are the administrative data on vaccination (10), information from the national seroprevalence 
survey of 2002 (11) and the demographic data on the age structure of population (12).

\section{Data on Vaccination within Administrative Control of Vaccination}

A two-dose measles vaccination schedule has been used between the years 2002 and 2015. The data on vaccination considered in this study are taken from the yearly reports of administrative controls of vaccination in Slovakia (10). These controls are based on personal inspections of the paediatricians' records performed by personnel of regional public health authorities. Administrative data on vaccination coverage were obtained from numbers of vaccinated and all children of the given age in the respective catchment area. The available data contain the 1st dose (MCV1, provided to children between 15-18 month of life), immunization coverage for cohorts born after 1992, and the 2nd dose (MCV2, provided to children in the 11th year of life), vaccination coverage for cohorts born after 1983. If the given cohort has been checked more than once, we have taken into consideration the most recent information, i.e. for the first dose (MCV1) in cohorts 1992-2003 vaccination coverage of 4-year-old children, and in cohorts 2004-2012 vaccination coverage of 3-year-old children. Similarly, we have considered for the second dose (MCV2) in cohorts 1983-1993 vaccination coverage of 11-year-old children, and in cohorts 1994-2003 vaccination coverage of 10-year-old children (Fig. 1).

\section{Seroprevalence Survey 2002}

The seroprevalence survey 2002 consisted of 3,640 individuals, among them 1,537 children up to 15 years and 2,103 subjects from the population older than 15 years (11).

Unfortunately, only aggregate data on seropositivity/negativity for particular age cohorts are available from the seroprevalence survey 2002.

For the purposes of this study we understand susceptible (S) to be the synonym to seronegative. Similarly, immune (resistant/ recovered (R)) shall be equivalent to seropositive.



Fig. 1. Vaccination coverage in Slovakia from 1981 to 2012 with the first (MCV1) and second dose (MCV2) for each age cohort. Asterisks denote estimations.

\section{Demographic Data}

Data related to the age structure of population are taken from the database DataCube (12)

\section{Primary Vaccination Failure}

The primary vaccination failure (PVF) is defined as a failure of a vaccine: an individual fails to develop the immune response to the vaccination and thus remains susceptible. In our model, we assume that the probability of PVF is 0.05 (13). It means that the immune response develops in $95 \%$ of vaccinated previously susceptible individuals after one dose of vaccine.

\section{Secondary Vaccination Failure}

The secondary vaccination failure (SVF), or waning immunity, is accompanied by a decrease of protective antibodies over time. Although cellular immunity may create protection for particular individuals whose antibodies titres are not sufficiently high, we did not consider it in our modelling due to insufficient information.

We have estimated the level of SVF according to the model of Lee and Nokes (2). We assume that in each age cohort the concentration of IgG antibody against measles follows a log-normal distribution with geometric mean of the titers GMT(t). Without exposing to wild measles virus or further vaccination, we assume that geometric mean titer decreases exponentially with time, i.e:

$$
\operatorname{GMT}(\mathrm{t})=\operatorname{GMT}(0) \times \exp (-\mathrm{WR} \times \mathrm{t})
$$

where $t$ denotes the time following the vaccination and WR represents the waning rate $(2,3)$ (Fig. 2).

For purposes of this analysis we define a seropositive or an immune individual as the person whose level of the antibody titers is above the so called critical level $\mathrm{C}_{\text {crit }}$. Once the amount of antibodies falls below the threshold $\mathrm{C}_{\text {cit }}$ (a minimum antibody level for protection against measles), the individual becomes again susceptible.

Based on our assumptions, we can express the proportion of susceptible individuals at time $\mathrm{t}, \operatorname{SVF}(\mathrm{t})$, as:



Fig. 2. Evolution of geometric mean titers (GMT) according to the age in particular cohort.

The decline of antibodies titers relates only to those individuals, who have been previously seroconverted to vaccinated, i.e. to the (1 - PVF) of vaccinated population. 
$\operatorname{SVF}(\mathrm{t})=\mathrm{P}\left(\mathrm{x}<\ln \left(\mathrm{C}_{\text {crit }}\right)\right)=\frac{1}{\sqrt{2 \pi \mathrm{SD}}} \int_{-\infty}^{\ln \mathrm{C}_{\text {crit }}} \exp \left[-\frac{(\mathrm{x}-\ln (\mathrm{GMT}(\mathrm{t})))^{2}}{2 \mathrm{SD}^{2}}\right] \mathrm{dx}$

where $\mathrm{x}$ represents a normally distributed random variable with the mean $\ln (\mathrm{GMT}(\mathrm{t}))$ and the standard deviation of the logarithm of antibody titers SD (2). It is important to note here that a decline of antibodies relates only to those individuals, who have been previously seroconverted to vaccinated, i.e. to (1-PVF) vaccinated population.

According to our assumptions, SVF is nonzero at the time of vaccination. It is a consequence of the normal distribution assumption. However, in all our scenarios mentioned below, the value of SVF is negligible in comparison to the value of PVF (4) (Fig. 3). Moreover, when compared to PVF for the given age cohort, the value of SVF is increasing with the time since the last vaccination.

\section{Estimation of Susceptibility Level}

An estimation procedure of the susceptibility level depends on the available information for the particular age cohort. In case the complete immunization records are available (i.e. the vaccination rates and the time of the first and the second dose administration), we proceed according to the scheme depicted in Figure 4. If precise administrative information is not available for a certain age cohort, we consider data from the last national seroprevalence survey.

We assume that before administration of the first dose all subjects are susceptible. Concerning vaccination, three situations can occur:

- First, a child is vaccinated and creates a post-vaccination immunity. The probability associated with this state can be computed as MCV1×(1-PVF).

- The second case happens with the probability MCV1 $\times$ PVF and describes a situation when a child is vaccinated but his or her immune system is not responding.

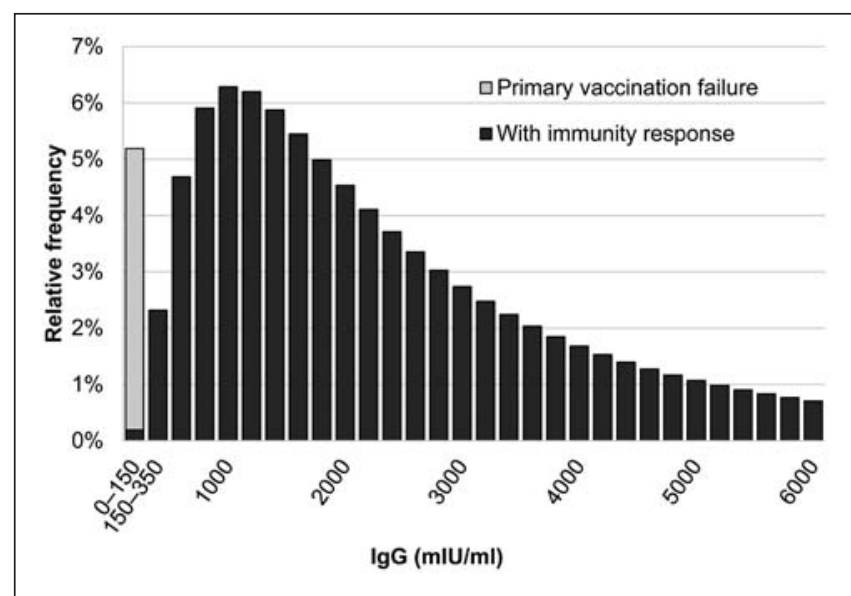

Fig. 3. Frequency distribution of the antibodies titers right after the administration of the first dose for the vaccinated population (model).

The leftmost column represents a group of seronegative individuals without immunity response to vaccination. The most of these cases are the result of the PVF (the grey area), others are the result of low immunity response to vaccination (the black area). The parameters of the lognormal distribution correspond to Scenarios 4 and 5 .

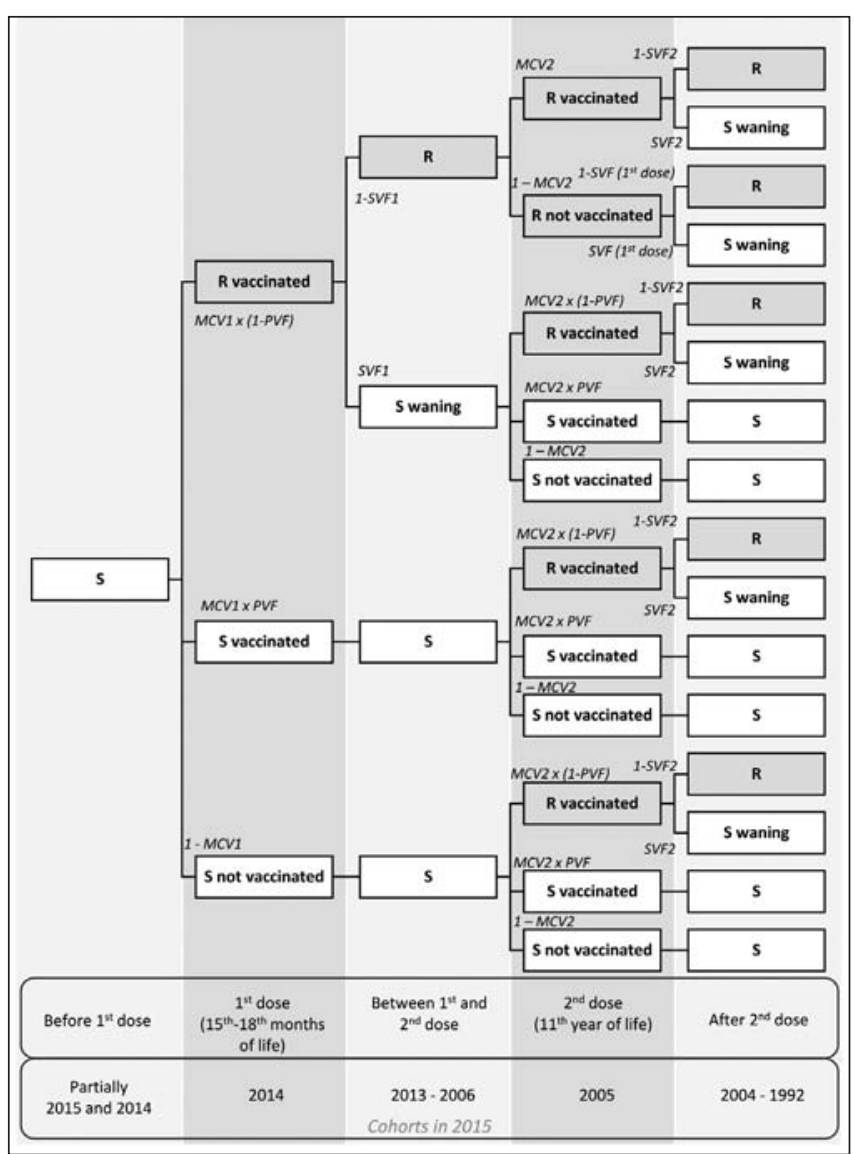

Fig. 4. Scheme of the calculation of proportions of susceptible/ immune individuals for the age cohorts with complete records of immunization history (time and vaccination rates for the first (MCV1) and second dose (MCV2)).

Susceptible and immune (recovered) individuals are represented by a symbol $\mathrm{S}$, and $\mathrm{R}$, respectively. The symbol PVF denotes the primary vaccination failure, SVF1 and SVF2 the secondary vaccination failure after the first and the second dose, respectively.

- If a child does not attend vaccination appointment at all, he or she remains susceptible. The associated probability of this case is (1-MCV1).

Under the current vaccination scheme the second dose of the MMR vaccine is administered in the 11th year of life. During the 9-year period between vaccinations, without the contact with measles virus, the status of the susceptible individual does not change. However, due to the waning immunity effect, some of the seropositive subjects might become susceptible again. Depending on the seroprevalence status of the particular individual and the action taken we determine individual's status after the administration of the second dose as follows:

- Naturally, immune individuals, who are vaccinated by the second dose, remain seropositive. We assume that the second dose acts as a booster, i.e. the level of antibodies jumps up again to the same level as it was after the first vaccination.

- If immune individuals do not attend the second vaccination appointment, they only stay immune if their level of antibodies has not declined below the protective boundary since the time of the first vaccination (i.e. the time of the SVF is calculated since the first vaccination).

- For the seronegative individuals the same three scenarios, mentioned above for the first vaccination, can occur. The 
only difference is that, in this case, we apply the level of MCV2 vaccination rates. Further, we assume that the values of PVF after the first and the second dose are equal and independent.

Several events relevant to our modelling have happened since 2002, when the state of the population susceptibility has been empirically estimated for the last time. First, the generation based shift should be considered. Compared to 2002, in 2015 the newly born cohorts of the years 2003-2015 have entered the population. On the other hand, the oldest cohorts, i.e. those born between the years 1902-1914, must be omitted from the 2015 estimation. Furthermore, according to the vaccination schedule between the years 2002-2015, the first dose has been administered to the cohorts born in the years 2001-2014 and the second dose has been given to the cohorts 1992-2005. Moreover, the effect of waning immunity can have a substantial impact on the prediction of susceptibility especially in the cohorts vaccinated the last time before the year 2002. Finally, the relative proportion of the cohorts, i.e. population age structure has changed due to population ageing and changes in the mortality rate.

\section{Model Inputs and Description of Scenarios}

A number of parameters entering the model were introduced in the previous section. Since the values of parameters cannot be estimated with certainty, we propose several possible scenarios for further analyses (Table 1).

In Scenario 1, we assume that the acquired antibodies ensure life-long immunity. Due to this assumption, this scenario can be considered as the most optimistic estimation of the number of susceptible individuals. The values of parameters in Scenarios 2 and 3 are taken from the relevant literature $(5,6)$. We consider only the longitudinal studies, which have provided the values of GMTs, mentioned the proportion of seronegative individuals in several time points and collected the data from at least 10 years. Because the parameters needed for our model were not reported elsewhere, we have estimated their values based on the published serological data. In order to avoid significant overestimation for the long-term period, we have only considered the GMT(t) values from the period longer than one year after vaccination. In Scenarios 4 and 5 the parameter values have been selected subject to expert appraisal. These values can be considered as a compromise among Scenario 1 and Scenarios 2 and 3.

\section{Description of Cohort Model}

\section{Cohorts Born before the Year 1968}

We assume that these birth cohorts acquired immunity to measles through natural infection, because measles infection was widespread prior to introduction of vaccination strategies. Since the values of GMT or the time of disease exposure are not available, we proposed to estimate the proportion of susceptible individuals in 2015 as follows:

$$
R(2015)=R(2002) \times(1-\operatorname{SVF}(2015-2002))
$$

where $\mathrm{R}(2015)$ and $\mathrm{R}(2002)$ are the proportion of seropositive individuals in respective years.

\section{Cohorts Born in the Years 1968-1982}

These cohorts were born after the introduction of routine measles vaccination and thus most likely had previously been vaccinated. However, no precise administrative data concerning vaccination rates, vaccine type or exact vaccination time were available. Moreover, some individuals might come to the contact with wild measles virus, which could influence the level of antibodies as well.

In our model, we set subjectively the proportion of PVF and vaccination omissions to $7 \%$. Let denote this group by $\mathrm{S}_{\mathrm{PVF}+\text { notvacc }}$. This group has been susceptible in the past (e.g. right at the time of vaccination) too. We assume that the rest of the observable seronegative individuals in 2002 was susceptible only due to waning immunity: this group was seropositive shortly after vaccination, but as the time passed, their antibodies have finally declined below the protection boundary.

Based on this assumption we express the level of geometric mean titer $\left(\mathrm{GMT}_{2002}\right)$ at the time of seroprevalence survey 2002 as:

$$
\begin{aligned}
& \mathrm{GMT}_{2002}=\exp \left(\operatorname{lnC} \mathrm{C}_{\text {crit }}-\mathrm{SD} \times \operatorname{norminv}\left(1-\mathrm{S}_{\mathrm{PVF}+\text { notvacc }}-\mathrm{R}(2002)\right.\right. \\
& \left.\left.\times\left(1-\mathrm{S}_{\mathrm{PVF}+\text { notvacc }}\right)\right)\right)
\end{aligned}
$$

where norminv is the inverse function of a standard cumulative normal distribution.

Consequently, we can determine the proportion of susceptible individuals in the year 2015 as follows:

$$
\mathrm{R}(2015)=\left(1-\mathrm{S}_{\mathrm{PVF}+\text { notvacc }}\right) \times(1-\mathrm{SVF}(2015-2002))
$$

In the calculation of SVF we set the value of geometric mean titer to

$$
\mathrm{GMT}_{2015}=\mathrm{GMT}_{2002} \times \exp ((2002-2015) \times \mathrm{WR})
$$

\section{Cohorts Born in the Years 1983-1991}

Administrative immunization records are partially available, therefore, it is possible to identify the year of the second dose administration (MCV2) for all birth cohorts in question. Moreover, since the last MMR shot has been given prior to the year 2002, a

Table 1. Parameter values of investigated scenarios

\begin{tabular}{|l|c|c|c|c|c|}
\hline & GMT(0) & SD & WR & C $_{\text {crit. }}$ & Ref. \\
\hline Scenario 1 & Without SVF, SVF = 0 & 0.92 & 0.069 year $^{-1}$ & $150 \mathrm{mlU} / \mathrm{mL}(350 \mathrm{mlU} / \mathrm{ml})$ & $(5)$ \\
\hline Scenario 2 & $1914 \mathrm{mlU} / \mathrm{mL}$ & 0.97 & 0.078 year $^{-1}$ & $120 \mathrm{mlU} / \mathrm{mL}$ & $(6)$ \\
\hline Scenario 3 & $1523 \mathrm{mlU} / \mathrm{mL}$ & 0.90 & 0.05 year $^{-1}$ & $150 \mathrm{mlU} / \mathrm{mL}(350 \mathrm{mlU} / \mathrm{ml})$ & estimated \\
\hline Scenario 4 & $2000 \mathrm{mlU} / \mathrm{mL}$ & 0.90 & 0.03 year ${ }^{-1}$ & $150 \mathrm{mlU} / \mathrm{mL}(350 \mathrm{mlU} / \mathrm{ml})$ & estimated \\
\hline Scenario 5 & $2000 \mathrm{mlU} / \mathrm{mL}$ & &
\end{tabular}


change in the seroprevalence between the years 2002 and 2015 is just a result of waning immunity.

The percentage of the susceptible individuals in the year 2015 has been estimated in two steps. First, using the available seroprevalence data 2002, we determine the proportion of susceptible individuals immediately after administration of the second dose. If we denote the time passed since the second vaccination by $\mathrm{t}_{\mathrm{MCV} 2}$ then, based on our assumptions, the proportion of seropositive individuals in the year 2002 equals to:

$$
\mathrm{R}(2002)=\left(1-\mathrm{S}_{\mathrm{PVF}+\text { notvacc }}\right) \times\left(1-\mathrm{SVF}\left(2002-\mathrm{t}_{\mathrm{MCV} 2}\right)\right)
$$

By rearranging the terms, we can express the proportion of susceptible individuals immediately after the administration of the second dose as follows:

$$
\mathrm{S}_{\mathrm{PVF}+\text { notvacc }}=1-\frac{\mathrm{R}(2002)}{1-\mathrm{SVF}\left(2002-\mathrm{t}_{\mathrm{MCV} 2}\right)}
$$

In the second step, we consider waning immunity between the years 2002 and 2015. Thus, the final estimate of the seropositive individuals, R(2015), can be determined by the following statement:

$$
\mathrm{R}(2015)=\left(1-\mathrm{S}_{\mathrm{PVF}+\text { notvacc }}\right) \times\left(1-\mathrm{SVF}\left(2015-\mathrm{t}_{\mathrm{MCV} 2}\right)\right)
$$

\section{Cohorts Born in the Years 1992-2003 (2004, 2005)}

The complete immunization records are available for all these birth cohorts. For the purposes of this analysis we have collected the vaccination rates and the time of the first and the second dose administration at the national level. This data let us to estimate the proportion of the susceptible individuals according to the scheme depicted in Fig. 4. In the year 2015, the birth cohorts 2004 and 2005 have just been vaccinated by the second dose of MMR vaccine. Therefore, at the time of preparation of this paper the final vaccination rates have not been published yet. Due to the previous development of vaccination rates we expect them to be approximately $97.5 \%$.

\section{Cohorts Born in the Years 2006-2012 (2013)}

According to the vaccination schedule until the year 2015 these cohorts have been vaccinated just with the first dose of MMR vaccine. To provide an estimation of a percentage of susceptible individuals in 2015 again, we have proceeded as outlined in the scheme in Fig. 4. At the time of preparation of this paper the final vaccination rate for the cohort born in 2013 has not been published yet. From the previous development of vaccination rates we expect it to be approximately $95 \%$.

\section{Cohort Born in the Year 2014}

In the year 2015, the birth cohort 2014 has already been partly vaccinated. Under the assumption of uniform population growth, we can propose that a quarter of the children is in age of 12-15 months. According to the current vaccination schedule this subgroup has not been vaccinated yet. Therefore, we consider all of them to be susceptible against measles $(16,17)$.

Another quarter of the children is in age of 15-18 months, i.e. they were exactly at the age of the first immunization. For the sake of simplicity, we assume that a half of these children has already developed antibodies, i.e. they are seropositive. Children aged 18-24 months already ought to be vaccinated. However, in practice, the first immunization is often delayed due to contraindications or precautions. Therefore, we assume that in this subgroup there are approximately $85 \%$ of seropositive children. Altogether, in the birth cohort 2014 the estimated proportion of the susceptible individuals was $45 \%$.

A similar estimation procedure applies to the birth cohort 2001 in the year 2002.

\section{Cohort Born in the Year 2015}

The youngest birth cohort consisted of infants. Due to potential protection by maternal antibodies we assume that approximately $35 \%$ of the children are immune, the rest is susceptible $(16,17)$. The same estimation has been applied to the birth cohort 2002 in the year 2002.

\section{RESULTS}

\section{Estimation of Secondary Vaccination Failure}

In none of the studied scenarios modelling the 10 year period waning immunity affects the estimated proportion of the susceptible population (Fig. 5). A more significant impact can be expected for individuals whose antibodies are close to the protection boundary (Fig. 6). However, for the longer time horizon (i.e. in the next few decades), waning of antibodies can potentially cause a substantial move of immune individuals back to the susceptible compartment due to immunity loss. However, the key driver values of this process (such as the waning rate and the initial level of vaccine-induced antibodies) are not currently presented in the literature in a directly usable form. The proposed Scenarios 2 and 3 provide pessimistic estimations, while Scenarios 4 and 5 represent a more optimistic situation.

\section{Cohort Model}

According to the seroprevalence survey 2002, apart from children up to one year, the cohorts of 17, 19 and 20-35 years old individuals show the highest proportion of susceptibles. The differences between these particular cohorts are not significant: except the cohort of 19 years old individuals whose relative immunity has been estimated to just $89.3 \%$, the rest remained above $90 \%$ (11).

The prediction for the year 2015 indicates slightly varied results. If the effect of waning immunity is considered (all scenarios except Scenario 1), the differences in the susceptibility among individual age cohorts are more significant. The largest impact can be observed in the cohorts vaccinated longer time ago.

Under all analysed scenarios, the most risky cohorts are the unvaccinated children up to one year of age and the adults in cohorts of 30-45 years (Fig. 7). Moreover, these cohorts are the most numerous: considering the age structure of the Slovak population, in this age group there is the largest number of susceptible individuals compared to other age cohorts (Fig. 8). Scenario 1, which ignores the effect of waning immunity, estimates the average susceptibility to measles in the Slovak population to 4.6\% for the year 2015. Scenario 3 provides the most pessimistic prediction: based on the proposed parameter values, the upper level of the average population susceptibility is estimated to be as high as $10.7 \%$. 




Fig. 5. The proportion of seronegative individuals (i.e. those whose antibodies are below $C_{\text {crit }}$ ) as a function of age for a representative age cohort.

Both PVF and SVF are considered here.

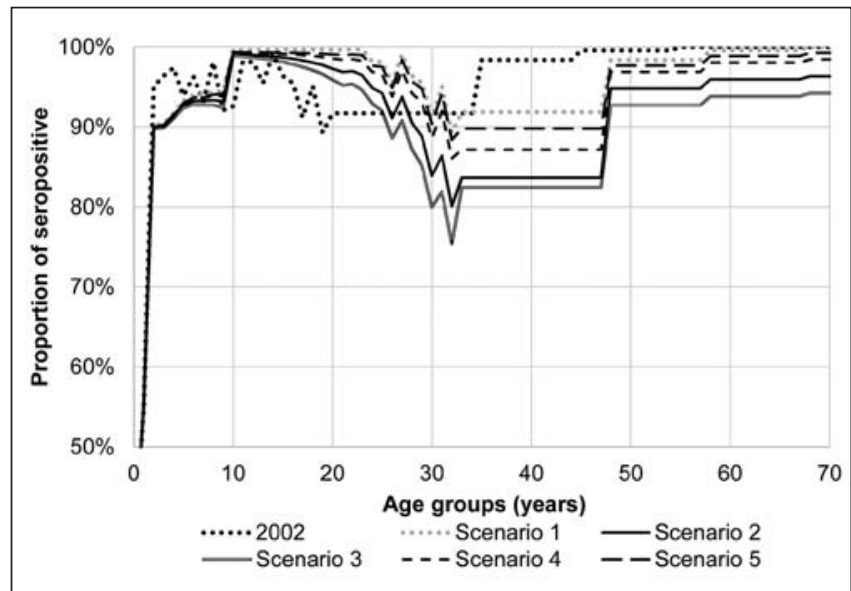

Fig. 7. The proportion of seropositive (immune) individuals in 2002 (seroprevalence survey data) and 2015 (estimated by the model).

\section{DISCUSSION}

Thanks to mandatory vaccination scheme the vaccination coverage against measles in Slovakia has stayed at a sufficiently high level for a long time. However, a decline in the yearly vaccination rate has currently been observed, mainly for the first dose of vaccine (MCV1). Decrease in vaccination coverage is most probably caused by anti-vaccination activities. Although nowadays such decline does not influence the average susceptibility in a significant manner, it might represent a threat for the future. A continuing decline in the yearly vaccination rate can lead to accumulation of the susceptible individuals in the younger cohorts and thus to a decrease in the total average susceptibility of the Slovak population.

A second recently recognized key determinant of the population susceptibility is a presence of waning immunity. Waning immunity becomes important especially in the situations when the spread of disease is interrupted or reduced: the individuals

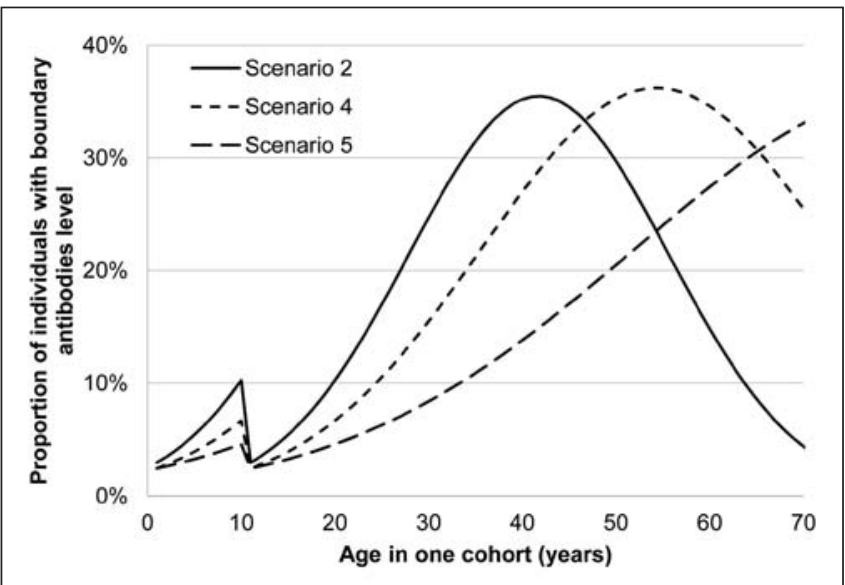

Fig. 6. The proportion of individuals with antibodies level close to the boundary of protection (i.e. with antibodies levels around 150-350 $\mathrm{m} / \mathrm{U} / \mathrm{ml})$ ) expressed as a function of age for one representative age cohort.

Scenario 3 is omitted due to the missing information on the upper critical level of antibodies.

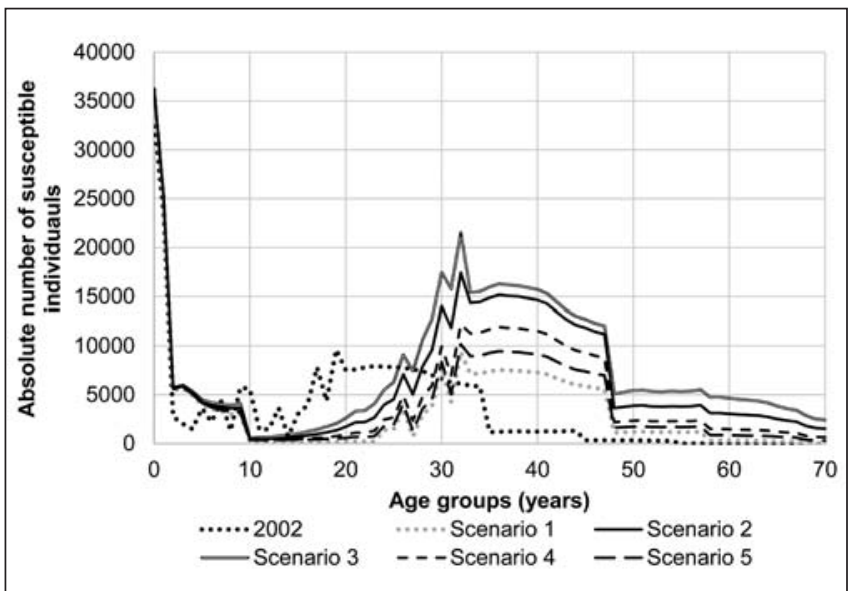

Fig. 8. The absolute numbers of susceptible individuals in the particular age cohorts in 2002 (seroprevalence survey data) and 2015 (estimated by the model).

have no contacts with the infection, and thus no natural immunity boosting applies to them (7). The latter describes the recent situation in Slovakia as during the last decade measles have appeared sporadically and only as imported cases.

Quantifying the process of waning immunity is not straightforward. The studies $(2,4,18)$ indicate that the decline in antibody levels shows exponential trend. This observation has also been supported by the study (7) in which the authors argue that the decline in antibody levels is more pronounced in individuals with high initial levels of antibodies in comparison to subjects with lower levels of antibodies.

The parameter values applied in our analysis have originated from the previous studies $(5,6)$. Although these studies do not directly describe the process of waning of antibody levels, from the presented results it is possible to identify the values of GMT(0), WR, and SD to a certain degree. It is important to note that only few longitudinal studies exist due to various obstacles in monitoring the same group of individuals for a long time period. 
Predictions based on the above mentioned studies $(5,6)$ (Scenario 2 and 3) seem to be too pessimistic even if values of GMT originating within the first year are not considered. The reason might lie in a relatively short observation period (10 and 15 years): estimation of the waning rate for a period longer than 30 years (the vaccination against measles was introduced in 1969) could be biased by the data of the shorter time period. Moreover, up-to-date published studies do not clearly identify whether the decrease in GMT is indeed exponential over long-term time periods, or the declining course of GMT is eventually stabilized at some level, or whether the decline could slow down after a certain period. In addition, it is largely unknown how cell-induced immunity affects susceptibility of the population (9). Thus, we have proposed to analyse Scenario 4 and 5 with more optimistic values of the model parameters.

Most relevant studies suggest that as the time from the last vaccination passes, the level of the IgG antibodies declines unless a contact with natural infection or further vaccination is present. However, a final quantification of the waning immunity process or the resulting impact on the immune status of individuals or population is not defined with certainty.

Since our model is strongly dependent on the above mentioned parameters which are not known exactly, any long-term prediction is unreliable.

Another estimation of the waning rate is presented in the paper by Mossong et al. (4). According to their findings the value of this parameter is approximately 0.056 per year (95\% CI: $0.033-0.077$ ). Unfortunately, the other values of the parameters are not presented in this study. Although the study is not based on a prospective design, the estimation can be considered as reliable.

Lee and Nokes (2) analysed the decline of the antibody level from a theoretical point of view. They introduced a mathematical model to predict a proportion of the seronegative individuals depending on various parameter setups. However, the values of waning rate $(0.173-0.347$ per year) seem to be implausibly high which results in unrealistic predictions. Although we have proceeded in a similar way we have decided to set up the values of parameters according to other published studies.

Our predictions via cohort model arise from information which is accessible for the particular age cohorts. The cohorts born before the year 1968 were most likely not vaccinated and highly probably they acquired immunity against measles through natural infection. Due to this fact, as the national seroprevalence study 2002 indicates, their immunity against measles is at a sufficiently high level. However, without further information we are not able either to identify whether waning immunity applies to these cohorts or to set up the values of the model parameters GMT(0), SD and WR. At least we can replace these values by those assigned to the currently vaccinated individuals. This approach probably results in a rather pessimistic prediction: according to the available seroprevalence surveys $(14,15)$, the antibodies titers of these cohorts are higher than those of recently vaccinated individuals. The reasons can lie not just in the higher initial level of the naturally induced antibodies but also in the lower waning rate.

For the next two groups of cohorts (born between the years 1968-1982 and 1983-1991) administrative immunization records are partially available. Our prediction of susceptibility of these cohorts is similar for both groups. The seroprevalence survey 2002 indicates that the immune status against measles for these age cohorts is not as favourable as for the older age cohorts. This observation can be explained by PVF, SVF or by individual omission of vaccination. In the modelling process we have considered these three main reasons separately in order to determine the value of SVF between the years 2002 and 2015. This may be seen as a limitation of the model, however, due to insufficient information we deem the used model as appropriate.

Except for the youngest cohorts, for the rest of cohorts the complete immunization records are available. These data are relevant enough to estimate the proportion of the susceptible individuals according to the theoretical scheme depicted in Fig. 4. Since now the estimation is based on more precise information on vaccination status of the particular cohorts, it could be regarded as more reliable. However, also these predictions might be biased to some degree due to missing information concerning e.g. the estimation of PVF.

The estimation of the susceptibility for one-year-old children is based on the subjective assumption of precise administration of the first dose. Due to lack of information, this approach can be regarded as sufficient.

According to the published studies, the susceptibility of children younger than one year depends on the acquired maternal antibodies (16). Contemporary, newborns of vaccinated mothers show much worse immunity against measles than children of mothers who have experienced natural infection $(16,17)$. In Slovakia, most of the current mothers had been previously vaccinated and just exceptionally came into contact with natural infection. Therefore, nowadays infants are at higher risk than those in the past.

A similar cohort model has been applied by Hens et al. (18). Based on the Belgium national seroprevalence study performed in 2006, the authors aimed to estimate susceptibility of the Belgium population against measles in the year 2013. Unlike our model, authors have assumed that with increasing time from the last vaccination the proportion of susceptible individuals rather than antibodies titres declines exponentially. Despite of different assumptions their conclusions correspond with our findings.

The objective of our study was to fill the gap in the missing estimates of the population susceptibility due to lack of seroprevalence survey in Slovakia in recent years. In 2013, the national seroprevalence survey was conducted in the neighbouring Czech Republic (15). Since the common past of these two countries and similar demographic trends, we have the unique opportunity to compare our results with their empirical findings, at least for older age cohorts. As the Czech Republic currently uses different vaccination schedule for measles than Slovakia, comparison of younger age cohorts is not appropriate. In the Czech Republic, the first dose is administered at 15 months of age and the second one 6-10 months after the first dose, i.e. approximately at 21-25 months of age. The Czech national survey has proved a significant decline of the antibodies titres during the first several years after vaccination. However, this decline does not seem to have a significant impact on the resulting proportion of susceptible individuals. On the other hand, a remarkable growth in the proportion of seronegative individuals has been present in the cohorts of 30-45 year olds. This observation is within the limits of our predictions. The decline of protection in these cohorts can be explained at least by two reasons. Firstly, most of these age groups were vaccinated between the years 1969 and 1984 when the vaccination, after its 
initiation, was being stabilized and primary vaccination failure was relatively high. Secondly, older cohorts were predominantly immunized by natural infection which provides better protection from a long-term aspect than vaccination.

Unfortunately, even the data reported in the current Czech seroprevalence survey are not sufficient to identify the proportion of PVF and SVF, and thus to estimate the effect of SVF on the future evolution of the population susceptibility.

The low level of seropositivity of several adult cohorts corresponds to the incidence of measles in the Czech Republic in 2014. A source of infection was a man who had imported the disease from India. A primary transmission had occurred among close contacts of the patient; afterwards the epidemics hit the medical staff. Cohorts born between the years 1970-1980 and unvaccinated youngest children were the most affected population groups. The transmission between vaccinated children and seniors occurred only sporadically (19).

Naturally, the next step of this research should be the prediction of the risk of spread of measles in Slovakia and the identification of the most risky cohorts. In this context it is necessary to consider the impact of waning immunity, especially for the individuals whose antibodies are close to the critical antibody level. As our results imply, the proportion of them may be significant especially among younger adults and thus among the potential parents of unvaccinated children. This issue is described in more detail in the studies by Glass and Grenfell, and Mossong et al. (3, 20). Although both papers deal mainly with theoretical aspects of waning immunity, it is possible to figure out from their conclusions that under certain conditions a spread of the disease can be significantly determined by individuals with antibodies titres near the boundary of protection.

The presented results of our analysis indicate that the herd immunity against measles in Slovakia is currently on a sufficiently high level. We have estimated the overall proportion of the immune individuals to be around the critical level needed to achieve the herd immunity, which is $95 \%$ for measles (1). However, there is a real threat that the future evolution of the population susceptibility will not be so favourable. As our study has indicated it depends on two key factors: trends in vaccination coverage and the potential effect of waning immunity. In this paper we have proposed a conceptual framework and a scheme for estimation of the state of susceptibility for particular age cohort. Nonetheless, it is important to identify input parameters out of a wisely conducted seroprevalence survey. Therefore, we suggest to the authorities to reintroduce a regular implementation of the national seroprevalence surveys.

\section{CONCLUSIONS}

The goal of this work was to analyse changes in the population susceptibility to measles during the last 13 years when the last national seroprevalence study was performed in Slovakia. We have integrated a theoretical model of waning immunity into the cohort model and thus estimated the susceptibility to measles of the Slovak population in the year 2015.

The model partially replaces missing seroprevalence survey, but the parameters of model and phenomenon of waning immunity are not exactly known. The variability and limited knowledge of the input parameters represent two sources of uncertainty about predicted susceptibility.

The most reliable source of data to estimate parameters would be a longitudinal study focused on monitoring antibody levels in the same individuals over a longer period of time. We are aware of the organizational and financial difficulties associated with such survey, so we suggest to reintroduce the regular implementation of the national seroprevalence survey in order to empirically determine the level of susceptibility (not only) for measles in Slovakia. Regular national seroprevalence surveys could solve the problem of lacking data and provide solid base for a reliable estimation of the proportion of susceptible individuals in the future and thus the risk of large-scale outbreaks.

\section{Acknowledgements}

The authors wish to extend their thanks to the Public Health Authority of the Slovak Republic, Department of Epidemiology, namely Mgr. Adriana Mečochová, for substantial assistance in collecting administrative data on vaccination in Slovakia.

\section{Funding}

The study was supported by the Slovak Research and Development Agency under No. APVV-0096-12 (EPIBIOMAT).

\section{Conflict of Interests}

None declared

\section{REFERENCES}

1. Strebel PM, Papania MJ, Fiebelkorn AP, Halsey Na. Measles vaccine. In: Plotkin SA, Orenstein W, Offit PA. Vaccines. 6th ed. Philadelphia, PA: Elsevier; 2013. p. 352-87.

2. Lee MS, Nokes DJ. Predicting and comparing long-term measles antibody profiles of different immunization policies. Bull World Health Organ. 2001;79(7):615-24.

3. Glass K, Grenfell BT. Antibody dynamics in childhood diseases: waning and boosting of immunity and the impact of vaccination. J Theor Biol. 2003 Mar 7;221(1):121-31.

4. Mossong J, O'Callaghan CJ, Ratnam S. Modelling antibody response to measles vaccine and subsequent waning of immunity in a low exposure population. Vaccine. 2000 Oct 15;19(4-5):523-9.

5. Davidkin I, Jokinen S, Broman M, Leinikki P, Peltola H. Persistence of measles, mumps, and rubella antibodies in an MMR-vaccinated cohort: a 20-year follow-up. J Infect Dis. 2008 Apr 1;197(7):950-6.

6. LeBaron CW, Beeler J, Sullivan BJ, Forghani B, Bi D, Beck C, et al. Persistence of measles antibodies after 2 doses of measles vaccine in a postelimination environment. Arch Pediatr Adolesc Med. 2007 Mar;161(3):294-301.

7. Kremer JR, Schneider F, Muller CP. Waning antibodies in measles and rubella vaccinees - a longitudinal study. Vaccine. 2006 Mar 24;24(14):2594601.

8. Kontio M, Jokinen S, Paunio M, Peltola H, Davidkin I. Waning antibody levels and avidity: implications for MMR vaccine-induced protection. J Infect Dis. 2012 Nov 15;206(10):1542-8.

9. Dine MS, Hutchins SS, Thomas A, Williams I, Bellini WJ, Redd SC. Persistence of vaccine-induced antibody to measles 26-33 years after vaccination. J Infect Dis. 2004 May 1;189 Suppl 1:S123-30.

10. Public Health Authority of the Slovak Republic. Annual reports of the Regional Public Health Authorities in the Slovak Republic [Internet]. Bratislava: Public Health Authority of the Slovak Republic [cited 2016 Feb14]. Available from: http://www.uvzsr.sk/index.php?option=com_co ntent\&view $=$ category\&layout=blog $\& i d=25 \&$ Itemid=34. (In Slovak.)

11. Public Health Authority of the Slovak Republic. The annual report of the Public Health Authority of the Slovak Republic for 2005 year [Internet]. 
Bratislava: Public Health Authority of the Slovak Republic; 2006. [cited 2016 Feb 14]. Available from: http://www.uvzsr.sk/docs/vs/vyrocna_sprava_uvz_sr_2005.pdf. (In Slovak.)

12. DATAcube [Internet]. Bratislava: Statistical Office of the Slovak Republic [cited 2016 Feb 14]. Available from: http://datacube.statistics.sk/ TM1WebSK/.

13. Uzicanin A, Zimmerman L. Field effectiveness of live attenuated measlescontaining vaccines: a review of published literature. J Infect Dis. 2011 Jul;204 Suppl 1:S133-48.

14. García-Comas L, Sanz Moreno JC, Ordobás Gavín M, Barranco Ordóñez D, García Gutiérrez J, Ramos Blázquez B, et al. Seroprevalence of measles and rubella virus antibodies in the population of the Community of Madrid, 2008-2009. J Infect Public Health. 2015 Sep-Oct;8(5):432-40.

15. Bílková-Fránková $H$, Kloudová A, Zelená H, Tomášková H, Šebáková H, Martinková I, et al. Multipurpose serological survey (measles, mumps, pertussis, viral hepatitis B): SS 2013, Czech Republic: final report. Zpr Cent Epidemiol Mikrobiol. 2014;23 Suppl 1:1-152. (In Czech.)

16. Leuridan E, Hens N, Hutse V, Ieven M, Aerts M, Van Damme P. Early waning of maternal measles antibodies in era of measles elimination: longitudinal study. BMJ. 2010 May 18;340:c1626. doi: 10.1136/bmj. c1626.
17. Plans $\mathrm{P}$, de Ory F, Campins M, Álvarez E, Payà $\mathrm{T}$, Guisasola E, et al. Prevalence of anti-rubella, anti-measles and anti-mumps IgG antibodies in neonates and pregnant women in Catalonia (Spain) in 2013: susceptibility to measles increased from 2003 to 2013. Eur J Clin Microbiol Infect Dis. 2015 Jun;34(6):1161-71

18. Hens N, Abrams S, Santermans E, Theeten H, Goeyvaerts N, Lernout $\mathrm{T}$, et al. Assessing the risk of measles resurgence in a highly vaccinated population: Belgium anno 2013. Euro Surveill. 2015 Jan 8;20(1). pii: 20998.

19. Trmal J, Kupcová J, Dvořáková L, Vaculíkova D, Limberková R, Šlajová I, et al. Return of measles in Ústí Region. Epidemiol Mikrobiol Imunol. 2014;63(2):154-9. (In Czech.)

20. Mossong J, Nokes DJ, Edmunds WJ, Cox MJ, Ratnam S, Muller CP. Modeling the impact of subclinical measles transmission in vaccinated populations with waning immunity. Am J Epidemiol. 1999 Dec 1;150(11):1238-49.

Received August 30, 2016 Accepted in revised formFebruary 6, 2017 